\title{
Cell signalling and the glutathione redox system
}

\author{
Giuseppe Filomeni $^{\mathrm{a},{ }^{*}}$, Giuseppe Rotilio ${ }^{\mathrm{a}}$, Maria Rosa Ciriolo ${ }^{\mathrm{b}}$ \\ a'Department of Biology, University of Rome "Tor Vergata", Via della Ricerca Scientifica, 00173 Rome, Italy \\ bDepartment of Biomedical Sciences, University of Chieti “G. D'Annunzio”, Via dei Vestini, 66100 Chieti, Italy
}

Received 5 March 2002; accepted 6 May 2002

\begin{abstract}
The reduction/oxidation (redox) state of the cell is a consequence of the balance between the levels of oxidising and reducing equivalents. A reducing intracellular environment is often associated with cell survival; however, redox unbalance is necessary since it represents a regulatory sensor for several nuclear transcription factors. Activator protein 1 (AP-1), nuclear factor- $\kappa \mathrm{B}(\mathrm{NF}-\kappa \mathrm{B})$ and protein tyrosine phosphatases 1-B (PTP-1B) are some of the well-known molecular factors for which a redox modulation of their activity has been demonstrated. The glutathione buffer system modulates cell response to redox changes induced by either external or intracellular stimuli. This paper summarises recent knowledge on the role played by several redox modulators in inducing signalling events that finally regulate cell cycle progression.
\end{abstract}

(C) 2002 Elsevier Science Inc. All rights reserved.

Keywords: Glutathione; Thiols; Redox regulation; Glutathione-S-thiolation; Cell signalling; Redox transcription factors

\section{Intracellular redox environment}

What does intracellular redox environment depend on? The intracellular redox state is determined by the contribution of different redox couples. Each couple can exchange electrons in such a way that, by giving or accepting reducing equivalents, may represent good cofactors in redox enzymatic reactions. The relative amount of reduced and oxidised form of each system may determine changes in cellular redox state by shifting this value towards oxidising or reducing conditions. The three most important redox systems we commonly find within the cells are: nicotinammide adenine dinucleotide phosphate (NADPH/ $\left.N A D P^{+}\right)$, thioredoxin $\left(T R X_{r e d} / T R X_{o x}\right)$ and glutathione (GSH/GSSG). Among these, the last one is the most important since glutathione concentration is about 500to 1000-fold higher than TRX and NADPH, so changes in reduced/oxidised glutathione buffer directly reflect intracellular redox alterations [1].

\footnotetext{
${ }^{*}$ Corresponding author. Tel.: +39-6-7259-4360; fax: +39-6-7259-4311. E-mail address: filomeni@bio.uniroma2.it (G. Filomeni).

Abbreviations: AP-1, activator protein 1; ASK-1, apoptosis signalregulating kinase; GSH, reduced glutathione; GSSG, oxidized glutathione; GS-R, glutathione mixed disulphide; GST, glutathione- $S$-transferase; $\gamma$ GCS, $\gamma$-glutamyl-cysteine synthetase; $\gamma$-GT, $\gamma$-glutamyl transpeptidase; JNK, c-jun $\mathrm{NH}_{2}$-terminal kinase; NF- $\mathrm{kB}$, nuclear factor- $\mathrm{\kappa B}$; PKC, protein kinase C; PTPase, protein tyrosine phosphatases; ROS, reactive oxygen species; TNF, tumour necrosis factor; TRX, thioredoxin.
}

\section{1. $N A D P H$}

Directly operating antioxidants limit the action of freely diffusing oxidising radicals, which alter redox environment, by scavenging them and re-reducing the oxidised biomolecules. In this context NADPH acts as hydrogen anion donor in several enzymatic processes such as the reduction of GSSG to GSH, catalysed by GSSG-reductase. Besides such cofactor role, recently has been suggested also for NADPH a direct action on reduction reactions, especially in mitochondrial compartment where it can scavenge toxic free radicals and repair biomoleculederived radicals. For a more detailed discussion of this topic we refer to [2].

\section{2. $T R X$}

Mammalian and prokaryotic TRXs are proteins with oxidoreductase activity [3]. So far, three distinct variants of human TRX, encoded by different genes, have been cloned and characterised in some detail: TRX-1, the most studied 12-kDa form of the TRX family; TRX-2, an isoenzyme located in mitochondria, which presents an additional 60 amino acids $\mathrm{NH}_{2}$-terminal mitochondrial translocation signal [4], and SpTRX, a variant highly expressed in spermatozoa [5]. TRX of all organisms, including the human TRX-1, -2 and SpTRX contain a highly conserved -Cys-Gly-Pro-Cys- active site, essential for the function 
as a general and potent protein disulphide oxidoreductase [6]. Specific protein disulphide targets for reduction by TRX are ribonucleotide reductase [7], protein disulphide isomerase [8] and several transcription factors including $\mathrm{p} 53, \mathrm{NF}-\kappa \mathrm{B}$ and AP-1. Furthermore, TRX is also a specific electron donor for many peroxiredoxins $[9,10]$, highly important for the reduction of peroxides. In addition, this small multifunctional protein can directly reduce some reactive oxygen species (ROS), as well as refold oxidised proteins, induce autocrine growth factor-like effects and cocytokine activity. The latter information is important in stimulating the recruitment and proliferation of lymphocytes and the growth of tumour cells [11].

\subsection{Glutathione}

Glutathione is the most abundant low molecular weight thiol inside mammalian cells. It is present as a reduced form (GSH) and two oxidised species: glutathione disulphide (GSSG) and glutathione mixed disulphide with protein thiols (GS-R) [12]. Both these forms can be catalytically reduced back to GSH by the NADPH-dependent glutathione reductase and TRX/glutaredoxin (GRX) system, respectively. Moreover, the nonenzymatic interconversion between GSSG and GS-R can occur [1,12]. Besides its function as intracellular redox buffer, glutathione possesses several other roles. It can function as modulator of activity of thiol-dependent enzymes, which possess "critical" cysteine residues sensitive to redox changes $[13,14]$. GSH can also represent a cofactor for antioxidant enzymes such as GSH-peroxidase, a selenium containing enzyme involved in the reduction of many peroxides, especially membrane lipids peroxides formed upon oxidative insults [15]. A further role for GSH has been suggested in intracellular copper transport and detoxification. In fact, through the cysteine thiol group it can bind $\mathrm{Cu}^{2+}$ contributing to copper delivery to the apoprotein of copper enzymes $[16,17]$. Moreover, in doing so, GSH decreases the dangerous effect of free intracellular copper by impeding potentially toxic redox reactions among metals and oxygen (Fenton reaction). Finally, GSH may represent a storage for cysteine, which can be toxic when present at high concentration.

Under physiological conditions the reduced form of glutathione is 10- to 100-fold higher than oxidised form. Experimental evidence of the last decade have demonstrated that changes in this ratio may govern several cellular reactions involved in signal transduction, cell cycle regulation and many other cellular processes $[1,18,19]$. Upon the effects of oxidative stimuli GSH/GSSG ratio tends to decrease by rising the concentration of glutathione disulphide or decreasing the reduced glutathione amount. However, in response to oxidative stress, cells maintain glutathione redox state through different mechanisms. Glutathione reductase activity should be increased, or GSSG excess, formed upon oxidative stress, should be extruded. Concomitantly, $\gamma$-GCS can be activated to produce newly synthesised glutathione, or thiol disulphide exchange can be promoted. Only when oxidative stress becomes prolonged, and cellular systems are no more able to counteract the ROS-mediated insults, the amount of free GSH decreases leading to irreversible cell degeneration and death.

\section{Glutathione, thiol redox state and the regulation of cellular processes}

Several evidence of the last few years supported the idea that GSH decrease is often associated with the apoptotic program. In our laboratory we demonstrated the fundamental role that GSH plays in the commitment of programmed cell death [20-22]. In fact, we evidenced that, upon apoptotic stimulus, cytochrome $c$ release from mitochondria occurs in concomitance with a massive extrusion of GSH through specific membrane translocators [20]. This phenomenon seemed to be one of the first event necessary to trigger cell death. In fact, by restoring the intracellular GSH content through the reversible inhibition of its extrusion by L-metionine or sulphobromo-phtalein we were able to revert apoptosis [21]. On the other hand, GSH lossdependent cytochrome $c$ release does not necessarily commit cell to death. Indeed, by depleting intracellular GSH with buthionine sulphoximine, a specific inhibitor of glutathione synthesis, we were able to detect cytosolic cytochrome $c$ but not the activation of executor caspases [22]. Moreover, inducing apoptosis by a thiol reducing compound, such as dithiotreitol, neither GSH extrusion nor cytochrome $c$ release from mitochondria was observed. Taken together, these results suggest a strong relationship between intracellular glutathione levels and mitochondriadependent apoptotic pathway; however, which are the link molecule(s) or the redox-sensitive steps that connect each other the two phenomena have still to be elucidated.

Chemical and physical stresses or ligand/receptor binding are some of the processes in which oxidative stress has been suggested to be the trigger factor of transduction pathways leading to apoptosis, proliferation and cell cycle regulation [23-25].

From the first evidence, suggesting ROS as mediators of cell signalling, several studies hypothesised a role for redox environment in the regulation of many different pathways. Growing data have proposed that ROS production occurred in association with the activation of several cellular processes such as proliferation, differentiation and death. Although signalling processes seemed to be modulated and largely explained by the activation of phosphorylative reactions cascade, the molecular mechanisms that link ROS production to these phenomena remain to be identified. Experimental evidence demonstrate that receptors tyrosine kinase, such as epidermal and platelet-derived growth factor receptors, can be activated by oxidising 
molecules resulting in activation of downstream signalling systems [26,27]. Furthermore, many protein tyrosine kinases are activated by oxidative stress. $\mathrm{H}_{2} \mathrm{O}_{2}$ and pervanadate induce tyrosine phosphorylation of Lck [28,29] and Fyn [29], but, although oxidative stress apparently stimulates many protein tyrosine kinases in cells, there is no evidence, so far, for a direct activation.

The association between oxidising agents and the phosphorylative events appears to be stronger for the serine/ threonine kinase family. Recent studies demonstrate that $\mathrm{H}_{2} \mathrm{O}_{2}$ leads to the activation of protein kinase $\mathrm{B}$ (PKB/Akt) and its association with Hsp27 [30]. PKC as well, seems to be activated by $\mathrm{H}_{2} \mathrm{O}_{2}$ and redox cycling quinones [31]. Moreover, a study performed on purified PKC showed that a selective oxidation at the $\mathrm{NH}_{2}$-terminal regulatory domain, which contain a zinc-thiolate cluster, led to the activated form of the enzyme [32]. On the contrary, oxidative modification at the $\mathrm{COOH}$-terminal catalytic domain resulted in a complete inactivation of PKC [33]. Moreover, it should be noted that serine/threonine phosphatases as well as protein tyrosine phosphatases (PTPases; described later), are regulated by redox changes. Studies in in vivo systems have demonstrated that thiol oxidation of protein phosphatases 1 and $2 \mathrm{~A}$ inhibited their enzymatic activity [34]. Furthermore, superoxide dismutase 1 (SOD1) prevents calcineurin-a serine/threonine phospatase which contains $\mathrm{Fe}$ and $\mathrm{Zn}$ in its active sitefrom inactivation mediated by oxidative damage both in vivo and in vitro [35].

Processes that involve oxidative changes and intracellular thiols modification have been recently associated to the modulation of gene expression. As mentioned before, the primary target of redox regulation can be the sulphydryl group of cysteine residue which, depending on the surrounding environment, could be easily oxidised to form a disulphide bond, sulphenic $(\mathrm{R}-\mathrm{SOH})$, sulphinic $\left(\mathrm{R}-\mathrm{SO}_{2} \mathrm{H}\right)$, or sulphonic $\left(\mathrm{R}-\mathrm{SO}_{3} \mathrm{H}\right)$ acid [13]. The last two oxidative modifications are irreversible, therefore, proteins that posses "reactive" cysteines-readily oxidised at physiological $\mathrm{pH}$ - should be more sensitive to oxidative stress, which may result in irreversible modifications of protein structure and function. Sulphenic acid, instead, can be either reduced to a thiol form or transformed in a disulphide bridge, a chemical specie which can be reduced back through the reaction catalysed by TRX and GRX. Apparently, one of the major mechanisms by which an oxidising environment leads to these effects is the increase of the intracellular "disulphide potential", including all those events that could oxidatively modify specific cysteine residues.

\subsection{Protein/protein disulphide bond formation}

Cysteines have been implicated in the response to altered redox potential and generation of ROS by the formation of intra and intermolecular disulphide bonds. Such changes have been demonstrated to occur in different redox-sensi- tive proteins leading to both "gain" or "loss" of function. In particular, while intramolecular disulphide bond formation results in alteration of protein structure and conformation, the intermolecular disulphide bridge could lead to dimers and/or multimers formation. The latter process may represent a regulatory pathway that potentially influence the association between different cellular proteins [36].

The $\gamma$-GCS belongs to the class of proteins that "sense" oxidising conditions, as a regulatory mechanism; thus, through reversible disulphide bonds formation the enzyme undergoes a gain of function [37]. The holoenzyme is formed by a $70 \mathrm{kDa}$ catalytic subunit ( $\gamma$-GCSc or Glclc) and a $27 \mathrm{kDa}$ regulatory subunit $(\gamma-\mathrm{GCSr}$ or Glclr). Although both subunits present several cysteine residues, two disulphide bonds and a thiol cysteine residue at the active site are required for optimal enzyme activity. One disulphide bond is intermolecular and it is necessary to link the two subunits; the other is intramolecular and does not affect the active site [38]. Oxidising conditions that result in GSH depletion promote formation of the intermolecular disulphide. This produces a conformational change increasing the affinity and the specificity of the glutamate binding site. Physiological GSH concentration, on the other hand, reduces the intermolecular disulphide, producing changes in conformation that favours GSH feedback inhibition [37].

Among proteins which, instead, are negatively regulated in their function upon formation of a disulphide bond, TRX and GSTp represent well-established examples. Studies, performed using the yeast two-hybrid system, led to identify TRX as an ASK-1 interacting regulatory protein [39]. The TRX/ASK-1 association gives rise to the inactivation of kinase activity of ASK-1; moreover, this interaction is found only in nonstressed cells and seems to be modulated by intracellular ROS levels. In particular, increase in ROS concentration causes disulphide bridge formation between TRX molecules and, as a result, ASK-1 can undergo multimerisation, which correspond to the active form of the enzyme [40]. ASK-1 has been suggested to activate p38- and JNK-upstream kinases, MKK3/6 and MKK4/7, respectively [41]. Signal transduction mediated by the phosphorylation cascade has to be tightly regulated because, once induced, it leads to the activation of different substrates, including several transcription factors involved in cell response to stress and damage (such as ATF-2, c-jun and p53) $[42,43]$. One example of such regulation could be represented by $\mathrm{JNK}$, the activity of which is maintained at low levels within the cell, even in the presence of high concentrations of growth factors. As described for ASK-1, JNK activity seems to be inhibited in nonstressed cells. Adler et al. [44] purified the inhibitory component of JNK and identified it as GSTp. Under resting conditions, JNK phosphorylation is inhibited upon its association with GSTp, while under $\mathrm{H}_{2} \mathrm{O}_{2}$ or UV treatment, the GSTp/ JNK complex dissociates leading to GSTp dimer and/or multimer structures formation and JNK activation [36]. 
The identification of TRX and GSTp proteins as modulators of JNK and ASK-1 activity, together with the knowledge that they are also modulators of intracellular GSH levels, provides a potential molecular mechanism through which redox-mediated signalling events could be linked to downstream processes mediated by stress kinases activation.

\subsection{Protein/glutathione mixed disulphide bond formation}

Oxidative alteration of a "reactive" cysteine usually leads to the formation of sulphenic derivative that in the presence of $\mathrm{GSH}$, is efficiently transformed in a mixed disulphide bond (GS-R). Therefore, glutathione- $S$-thiolation represents a first line of defence against oxidative stress that, instead results in irreversible protein thiol oxidation of cysteines to sulphinic or sulphonic acid. Recently, it has been demonstrate that the process of glutathione- $S$-thiolation is also involved in the regulation (activation/deactivation) of protein activity during free radicals-mediated oxidative reactions $[1,14]$. Among cellular proteins, that appear to be modulated by this redox- sensitive process, transcription factors are included. In fact, in vitro experiments have demonstrated that transcription factors can be inhibited in their DNA binding activity through the $S$-thiolation of specific cysteines. The most important evidence derives from studies performed by Klatt et al. [45] who demonstrated that in vitro transcriptional activity of AP-1 is regulated by the redox state of a specific cysteine placed on the interface between the two cjun subunits. Moreover, a similar regulation has been

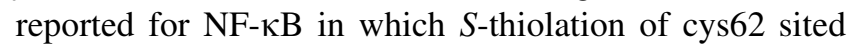
at the p50 subunit of the dimer has been demonstrated [46] in addition to the well-established activation of this transcription factor by a phosphorylative process. In fact, the activation of NF- $\mathrm{KB}$ relies on dissociation of an inhibitory

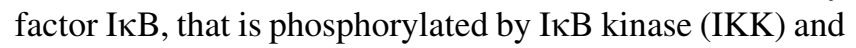
targeted for the ubiquitination/degradation pathway. Therefore, as shown in Fig. 1, a dual redox regulation of cellular signalling by modulation of transcription factors can be suggested: (1) Indirect induction of transcription factors by oxidant-mediated activation of phosphorylative events; (2) direct oxidative changes of reactive cysteines that inhibits DNA binding events.

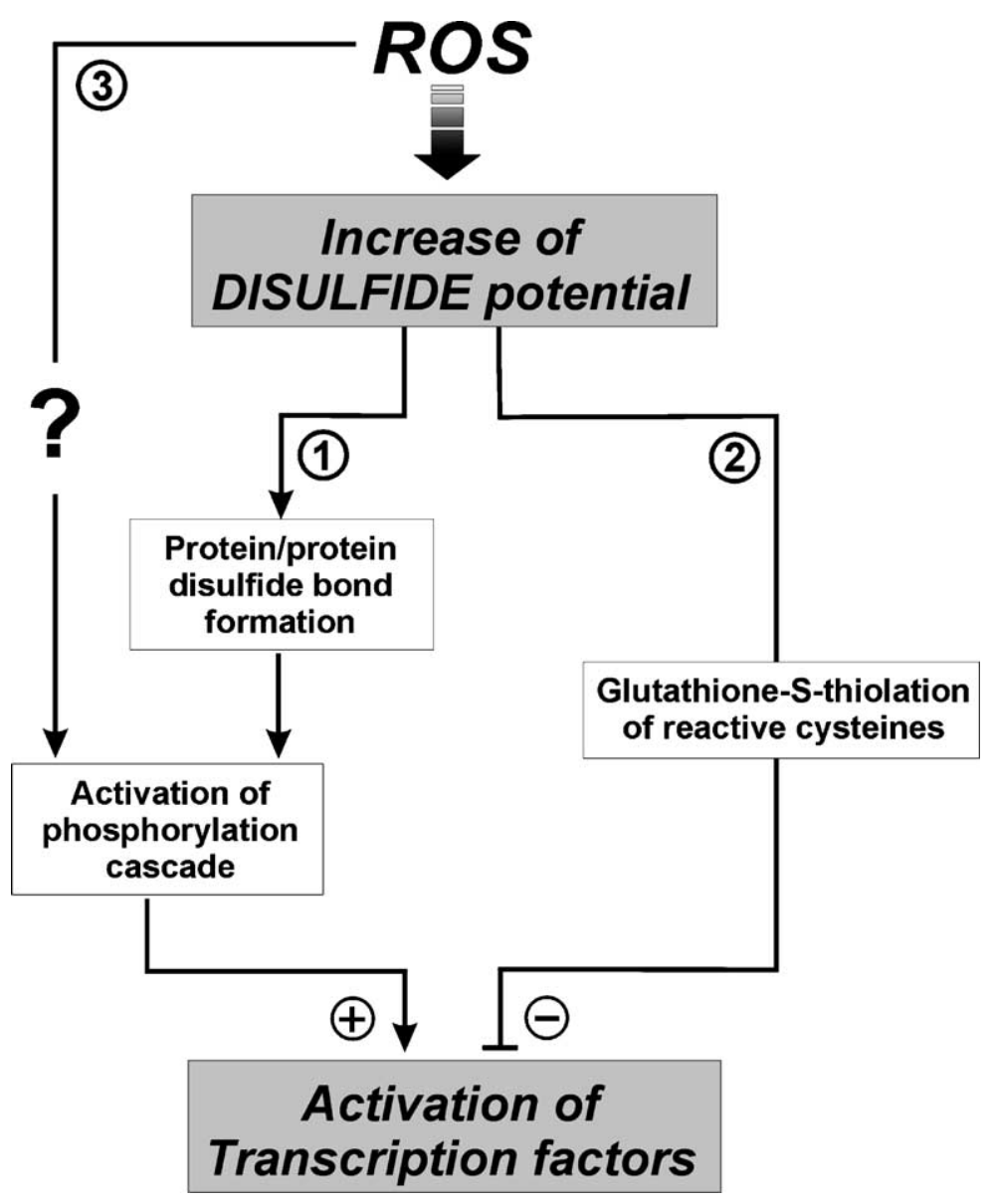

Fig. 1. Proposed pathways for oxidant-mediated signal transduction. ROS can raise "disulphide potential" of cellular environment resulting either in protein/ protein disulphide bond formation between regulatory proteins (TRX, GSTp) (1), or in the $S$-glutathiolation of "reactive" cysteines belonging to transcription factors (2). The first pathway indirectly leads to the activation of the final substrates through the induction of phosphorylative reactions catalysed by protein kinases (ASK-1, JNK). The second pathway directly inhibits the capability of transcription factors to bind DNA. A further linkage between oxidative burst and cellular signalling is represented by the still unclear association among ROS and phosphorylative events (3), as evidenced for receptor tyrosine kinases. 
Unfortunately, there is no evidence for an in vivo redox regulation of transcription factors, in particular, whether changes of protein thiols redox state are positively or negatively related to the transcriptional activities. However, evidence supporting the hypothesis for a transcriptional modulation in cultured cell systems is growing. Wu et al. [47] demonstrated that, upon oxidative stimuli p53 is oxidised to a mixed disulphide with GSH and that this modification was able to inhibit its transcriptional activity and its nuclear localisation. Furthermore, PTPase activity is highly regulated by oxidation/reduction reactions involving the cysteine required for catalysis [48,49]. PTPases share a conserved 230 amino acid domain containing the cysteine residue that catalyses the hydrolysis of phosphate from phospho-tyrosine residues by the formation of a cysteinyl-phosphate intermediate. PTPases are involved in the regulation of reversible tyrosine phosphorylation in the insulin action pathway. Recently, it has been established that insulin stimulation generates a burst of intracellular $\mathrm{H}_{2} \mathrm{O}_{2}$ in insulin-sensitive cells, which is associated with reversible oxidative inhibition of overall cellular PTPase activity, especially the PTP-1B family member [50].

On the basis of these findings, the question to which researchers are trying to answer is whether protein thiol redox state may represent a "molecular switch" able to reversibly activate/deactivate protein function: a form of modulation comparable to the well-established phosphorylative regulation mode by which the addition of a phosphate group dramatically changes protein activity.

\section{Extracellular environment and intracellular redox response}

The extracellular environment can dialogue with the intracellular compartments in a wide range of modalities. External molecules such as lipophylic hormones or esterified compounds, can freely cross the plasma membrane owing to their chemical nature and act inside the cell without restrictions. On the other hand, hydrophylic molecules can enter the cell only through the action of specific channels or ATP-dependent pumps. Finally, there are compounds which are totally membrane-impermeable but their presence can stimulate a cell response by the action of specific receptors that transduce the signal into the cytosol. As a result, each molecule represents both a marker of the extracellular milieu and an input to which cell responds (Fig. 2).

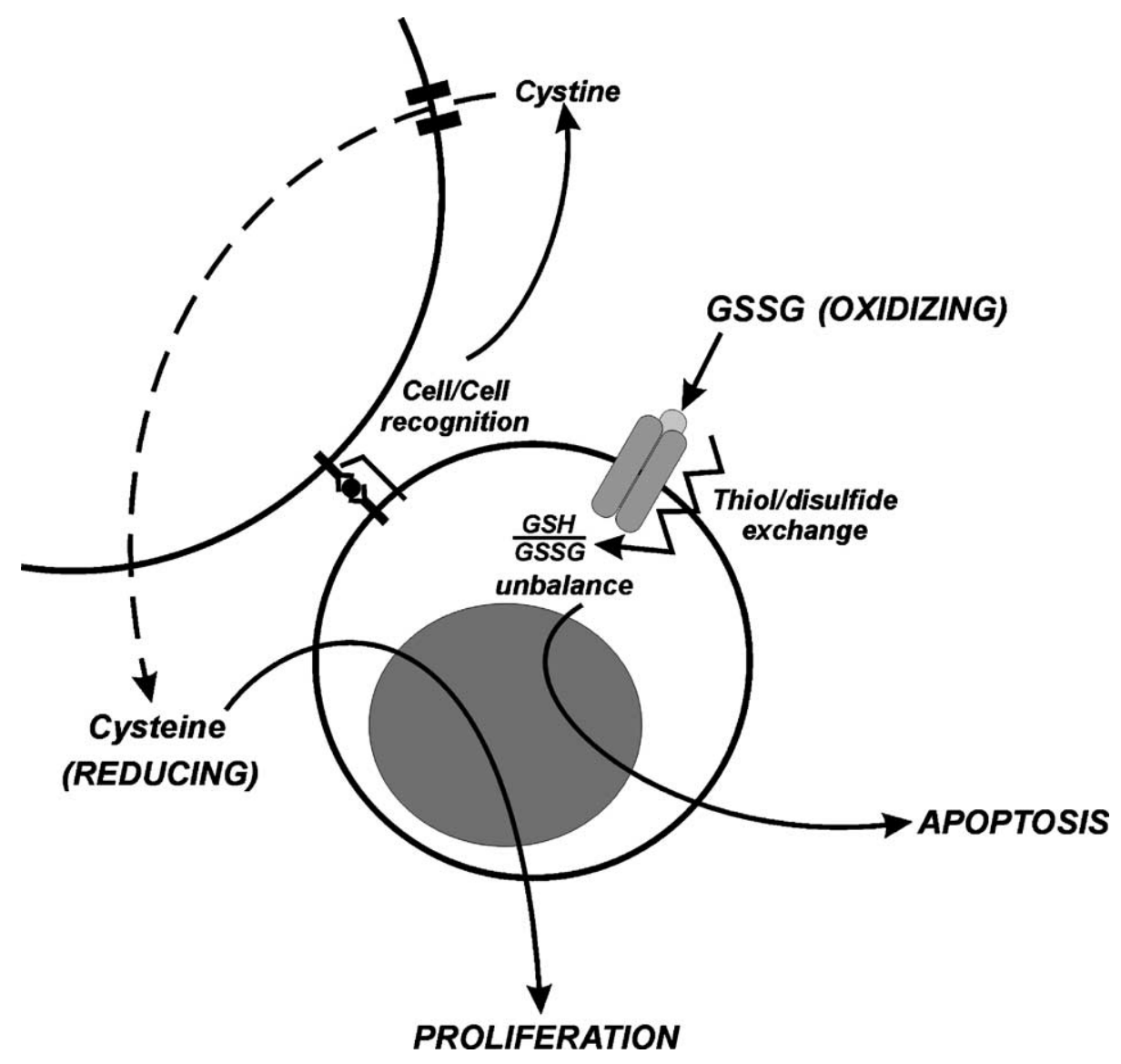

Fig. 2. A possible scheme for mechanisms by which the extracellular milieu indirectly induces changes in the intracellular redox environment. The nature of external molecules (reducing or oxidising) and of the cell involved lead to activation of different pathways, which eventually result in life (proliferation) or death (apoptosis). 
Reducing or oxidising molecules, such as TRX and/or $\mathrm{H}_{2} \mathrm{O}_{2}$, can be present outside the cell thus changing extracellular redox milieu. Furthermore, $\mathrm{H}_{2} \mathrm{O}_{2}$ can cross the plasma membrane and enter the cells leading to changes in the intracellular redox environment. Many membrane proteins can produce $\mathrm{H}_{2} \mathrm{O}_{2}$ upon different stimuli. For instance, the NADPH oxidase expressed on neutrophil membrane generates high levels of $\mathrm{H}_{2} \mathrm{O}_{2}$ as a defence mechanism in response to bacterial attack [51]. However, besides this "nonfutile" generation of oxidant species, as showed during an inflammatory response or upon the binding of insulin to its receptor, cells may produce lower amount of $\mathrm{H}_{2} \mathrm{O}_{2}$ as byproduct of enzyme reactions such as that catalysed by membrane $\gamma$-GT [52]. This enzyme is essential for GSH uptake since the reduced tripeptide per se is unable to cross the plasma membrane but it must be cleaved. In this reaction an oxidative rupture of the peptide bond between glutamic acid and cysteinylglycine occurs, and $\mathrm{H}_{2} \mathrm{O}_{2}$, which may act on intracellular redox-sensitive molecular factors, is produced. It has been established that the antitumour activity exerted by GSH on human ovarian carcinoma cells as well as the prevention of apoptosis and maintenance of proliferation in U937 cells is mediated by $\gamma$-GT-produced $\mathrm{H}_{2} \mathrm{O}_{2}[53,54]$. In fact, as a consequence of $\mathrm{H}_{2} \mathrm{O}_{2}$ generation, NF- $\mathrm{KB}$ is activated and many downstream genes, such as manganese containing SOD (Mn SOD) and $\gamma$-GCS, are actively transcribed as response to cellular redox changes. It is interesting to note that protein kinases are also involved in cell response mediated by several ligands, such as TNF superfamily. In fact, TNF/TNF receptor binding-as mentioned for insulin-has been shown to be associated with $\mathrm{H}_{2} \mathrm{O}_{2}$ generation, protein $S$-glutathiolation induction [55], and protein/protein disulphide bond formation [36]. As a consequence, such oxidative changes may, in turn, amplify the TNF receptor-mediated signal. Taking into account that oxidative alteration can function either as activator of upstream protein kinases, such as stress activated protein kinase (SAPK), extracellular signal regulated kinase (ERK) 1, 2, JNK and p38 [56], or inhibitor of downstream transcription factors, such as AP-1 and NF- $\mathrm{KB}$, the decision between life and death depends on the relative strength of the oxidative stress and on the type of cell involved.

Redox-active molecules, such as dithiotreitol, diamide and $\mathrm{H}_{2} \mathrm{O}_{2}$ directly alter intracellular environment by entering the cell and acting on target proteins. On the basis of the well-established knowledge on cell response upon ligand/ receptor binding - a mechanism that can produce the activation of several processes without a direct communication with the inside of the cell-the question which we have tried to answer was: "Could impermeable redoxactive molecules be able to change intracellular redox environment without crossing the plasma membrane?"

Previous work performed on isolated erythrocytes established that intracellular GSH content could be modulated by external molecules. Reglinski et al. [57] demonstrated that by adding increasing concentrations of the Ellmann's reagent to red blood cells, intracellular GSH levels decreased in a dose dependent manner. Moreover, spinecho NMR analyses suggested that oxidative events led to the formation of mixed disulphides. Few years later, our laboratory evidenced that the phenomenon previously described could be reverted by giving reduced glutathione to erythrocytes. This induced an increase in intracellular GSH although no external GSH crossed the red cell membrane [58]. The mechanism through which these phenomena were explained, was a thiol/disulphide exchange among thiol residues of proteins spanning across the plasma membrane. These studies suggested an involvement of both hexose transport protein and spectrin as targets of these redox reactions. In particular, Ellmann's reagent should block exofacial cysteine residues of hexose transporter determining an increase of intracellular glutathione mixed disulphides with spectrin thiols [57]. Therefore, thiol/disulphide exchange could represent a novel mode for transducing signals from extracellular milieu to intracellular environment.

From this point of view, thiol-containing compounds could represent signal molecules when act on specific transmembrane proteins. On the other hand, cysteine-rich membrane proteins could represent putative receptors, especially if they are known to be involved in the activation of some well-established signal transduction pathways. The cysteine-rich TNF receptors superfamily is a good example of what we have hypothesised. Even if interactions between redox-active molecules and these receptors are probably not so efficient as that mediated by binding of physiological effectors, the structure and the function of membrane receptors may be modified by reaction with these compounds in such a way as to start a signalling pathway.

\section{Conclusion and hypotheses for future work}

Recently, we have tried to modulate the intracellular redox environment of eukaryotic cells by treating a human promonocytic cell line, U937, with glutathione disulphide. This compound is a nonpermeable agent usually present at very low concentration both inside and outside the cell, since it is efficiently reduced back to GSH by the activity of GSSG-reductase. However, under an oxidative burst, GSSG can be produced at high rate and it can be extruded from cells into the extracellular milieu, becoming a powerful pro-oxidising molecule.

GSSG was able to alter the intracellular redox-buffer of U937 cells in terms of reduced and oxidised glutathione and mixed disulphides. In particular, the GSSG treatment resulted in a significant decrement in intracellular GSH that was paralleled by a highly significant increase in the mixed disulphide form of GSH with intracellular protein thiols, without changes in the GSSG content [59]. These 
results are in agreement with the above mentioned data obtained by Reglinski et al. [57], and are not related to possible $\mathrm{H}_{2} \mathrm{O}_{2}$ generation upon $\gamma$-GT activity; in fact, GSSG is not a good substrate for this enzyme and acivicin, a specific inhibitor of $\gamma-\mathrm{GT}$, did not alter the GSSGinduced redox changes. Since GSSG is a nonpermeable compound, it has to exert its oxidative effects by oxidising specific residues on the external side of the cell. A disulphide/thiol exchange along a chain of cysteine residues of transmembrane proteins might be hypothesised. The oxidative unbalance, finally, leads to cell death by activation of the mitochondrial apoptotic machinery, moreover, due to the specificity of GSSG to be toxic only for tumour cells [59], we suggest that the target of its action may be represented by TRAIL receptors, the transmembrane proapoptotic receptors involved in tumour suppression [60].

Taken together, these findings may suggest a novel role for the extracellular redox milieu in cell signalling and viability, that crosstalks with cellular systems and induces cell response by a mechanism that does not necessary involve a direct action of redox species on intracellular targets. In particular, extracellular redox signals could act through secondary mediators, which may be represented either by transmembrane cysteine-rich receptors or by specialised cells. The latter hypothesis is supported by recent findings which demonstrated that antigen-presenting cells increase extracellular cysteine levels stimulating the proliferation of activated $T$ cells [61]. In fact, most of the extracellular cysteine equivalents, necessary for the activation of proliferation, exist as the oxidised form, cystine, which $\mathrm{T}$ cells are unable to be internalised. Antigen-presenting cells take up cystine from the extracellular milieu by using the $\mathrm{X}_{\mathrm{c}}{ }^{-}$transporter system, reduce cystine to cysteine and extrude cysteine into the extracellular space where it comes be taken up by T cells. Moreover, dendritic cells secrete TRX, which can directly convert extracellular cystine to cysteine $[61,62]$. In this way a further mechanism by which extracellular redox environment is involved in cell signalling is available.

\section{Acknowledgments}

This work was partially supported by MURST “Cofinanziamento 2000", CNR and Ministero della Sanità "Progetto di Ricerca Finalizzata".

\section{References}

[1] Schafer FQ, Buettner GR. Redox environment of the cell as viewed through the redox state of the glutathione disulfide/glutathione couple. Free Radic Biol Med 2001;30:1191-212.

[2] Kirsgh M, De Groot H. NAD(P)H, a directly operating antioxidant? FASEB J 2001;15:1569-74.

[3] Holmgren A. Thioredoxin. Annu Rev Biochem 1985;54:237-71.
[4] Spyrou G, Enmark E, Miranda-Vizuete A, Gustafsson JA. Cloning and expression of a novel mammalian thioredoxin. J Biol Chem 1997;276:2936-41.

[5] Miranda-Vizuete A, Ljiung J, Damdimopoulos A, Gustafsson JA, Oko R, Pelto-Huikko M, Spyrou G. Characterization of Sptrx, a novel member of the thioredoxin family specifically expressed in human spermatozoa. J Biol Chem 2001;276:31567-74.

[6] Holmgren A. Thioredoxin and glutaredoxin systems. J Biol Chem 1989;264:13963-6.

[7] Reichard P. From RNA to DNA, why so many ribonucleotide reductases? Science 1993;260:1773-7.

[8] Lundstrom J, Holmgen A. Protein disulfide-isomerase is a substrate for thioredoxin reductase and has thioredoxin-like activity. J Biol Chem 1990;265:9114-20.

[9] Chae HZ, Kang SW, Rhee SG. Isoforms of mammalian peroxiredoxin that reduce peroxides in presence of thioredoxin. Methods Enzymol 1999;300:219-26.

[10] Nordberg J, Arnér ESJ. Reactive oxygen species, antioxidants and mammalian thioredoxin system. Free Radic Biol Med 2001;31: 1287-312.

[11] Wakasugi N, Tagaya Y, Wakasugi H, Mitsui A, Maeda M, Yodoi J, Tursz T. Adult T-cell leukemia-derived factor/thioredoxin, produced by both human T-lymphotropic virus type I- and Epstein-Barr virustransformed lymphocytes, act as an autocrine growth factor and synergizes with interleukin 1 and interleukin 2. Proc Natl Acad Sci U S A 1990;87:8282-6.

[12] Meister A, Anderson ME. Glutathione. Annu Rev Biochem 1983;52:711-60.

[13] Finkel T. Redox-dependent signal transduction. FEBS Lett 2000;476:52-4

[14] Klatt P, Lamas S. Regulation of protein function by S-glutathiolation in response to oxidative and nitrosative stress. Eur J Biochem 2000;267:4928-44.

[15] Flohè L. Glutathione peroxidase: fact and fiction. Ciba Found Symp 1978;65:95-122.

[16] Ferreira AM, Ciriolo MR, Marcocci L, Rotilio G. Copper(I) transfer into metallothionein mediated by glutathione. Biochem J 1993;292:673-6.

[17] Ciriolo MR, Desideri A, Paci M, Rotilio G. Reconstitution of $\mathrm{Cu}, \mathrm{Zn}$ superoxide dismutase by the $\mathrm{Cu}(\mathrm{I})$-glutathione complex. J Biol Chem 1990;265:11030-4.

[18] Cotgreave IA, Gerdes RG. Recent trends in glutathione biochemistryglutathione-protein interactions: a molecular link between oxidative stress and cell proliferation? Biochem Biophys Res Commun 1998;242:1-9.

[19] Herrlich P, Bohmer FD. Redox regulation of signal transduction in mammalian cells. Biochem Pharmacol 2000;59:35-41.

[20] Ghibelli L, Coppola S, Rotilio G, Lafavia E, Maresca V, Ciriolo MR. Non-oxidative loss of glutathione in apoptosis via GSH extrusion. Biochem Biophys Res Commun 1995;216:313-20.

[21] Ghibelli L, Fanelli C, Rotilio G, Lafavia E, Coppola S, Colussi C, Civitareale P, Ciriolo MR. Rescue of cells from apoptosis by inhibition of active GSH extrusion. FASEB J 1998;12:479-86.

[22] Ghibelli L, Coppola S, Fanelli C, Rotilio G, Civitareale P, Scovassi AI, Ciriolo MR. Glutathione depletion causes cytochrome $\mathrm{c}$ release even in the absence of cell commitment to apoptosis. FASEB J 1999;13:2031-6.

[23] Buttke TM, Sandstrom PA. Oxidative stress as mediator of apoptosis. Immunol Today 1994;15:7-10.

[24] Schulze-Osthoff K, Ferrari D, Los M, Wesselborg S, Peter ME. Apoptosis signaling by death receptors. Eur J Biochem 1998;254: 439-59.

[25] Wang X, Martindale JL, Liu Y, Holbrook NJ. The cellular response to oxidative stress: influences of mitogen-activated protein kinase signalling pathways on cell survival. Biochem J 1998;333: 291-300. 
[26] Heldin CH. Dimerization of cell surface receptors in signal transduction. Cell 1995;80:213-23.

[27] Pawson T. Protein modules and signalling networks. Nature 1995;373:573-80.

[28] Hardwick JS, Sefton BM. The activated form of the Lck tyrosine protein kinase in cells exposed to hydrogen peroxide is phosphorylated at both Tyr-394 and Tyr-505. J Biol Chem 1997;272:25429-32.

[29] Schieven GL, Kirihara JM, Myers DE, Ledbetter JA, Uckun FM. Reactive oxygen intermediates activate NF-kappa B in a tyrosine kinase-dependent mechanism and in combination with vanadate activate the p56lck and p59fyn tyrosine kinases in human lymphocytes. Blood 1993;82:1212-20.

[30] Konishi H, Matsuzaki H, Tanaka M, Takemura Y, Kuroda S, Ono Y, Kikkawa U. Activation of protein kinase B (Akt/RAC-protein kinase) by cellular stress and its association with heat shock protein Hsp27. FEBS Lett 1997;410:493-8.

[31] Kass GE, Duddy SK, Orrenius S. Activation of hepatocyte protein kinase C by redox-cycling quinones. Biochem J 1989;260:499-507.

[32] Gopalakrishna R, Anderson WB. $\mathrm{Ca}^{2+}$ - and phospholipid-independent activation of protein kinase $\mathrm{C}$ by selective oxidative modification of the regulatory domain. Proc Natl Acad Sci U S A 1989;86:6758-62.

[33] Gopalakrishna R, Anderson WB. Susceptibility of protein kinase C to oxidative inactivation: loss of both phosphotransferase activity and phorbol diester binding. FEBS Lett 1987;225:233-7.

[34] Nemani R, Lee EY. Reactivity of sulfhydryl groups of the catalytic subunits of rabbit skeletal muscle protein phosphatases 1 and 2A. Arch Biochem Biophys 1993;300:24-9.

[35] Wang X, Culotta VC, Klee CB. Superoxide dismutase protects calcineurin from inactivation. Nature 1996;383:434-7.

[36] Adler V, Yin Z, Tew KD, Ronai Z. Role of redox potential and reactive oxygen species in stress signaling. Oncogene 1999;18:6104-11.

[37] Soltaninassab SR, Sekhar KR, Meredith MJ, Freeman ML. Multifaceted regulation of $\gamma$-glutamylcysteine synthetase. J Cell Physiol 2000;182:163-70.

[38] Tu Z, Anders MW. Identification of an important cysteine in human glutamate-cysteine ligase catalytic subunit by sited direct mutagenesis. Biochem J 1998;336:675-80.

[39] Saitoh M, Nishitoh H, Fujii M, Takeda K, Tobiume K, Sawada Y, Kawabata M, Miyazono K, Ichijo H. Mammalian thioredoxin is a direct inhibitor of apoptosis signal-regulating kinase (ASK) 1. EMBO J 1998;17:2596-606.

[40] Gotoh Y, Cooper JA. Reactive oxygen species- and dimerizationinduced activation of apoptosis signal-regulating kinase 1 in tumor necrosis factor-alpha signal transduction. J Biol Chem 1998;273:17477-82.

[41] Ichijo H, Nishida E, Irie K, Dijke P, Saitoh M, Moriguchi T, Takagi M, Matsumoto K, Miyzaono K, Gotoh Y. Induction of apoptosis by ASK1, a mammalian MAPKKK that activates SAPK/JNK and p38 signaling pathways. Science 1997;275:90-4.

[42] Ip YT, Davis RJ. Signal transduction by the c-Jun N-terminal kinase (JNK) - from inflammation to development. Curr Opin Cell Biol 1998;10:205-19.

[43] Adler V, Pincus MR, Miramoto T, Fuchs SY, Bluth MJ, Brandt-Rauf PW, Friedman FK, Robinson RC, Chen JM, Wang XW, Harris CC, Ronai Z. Conformation-dependent phosphorylation of p53. Proc Natl Acad Sci U S A 1997;94:1686-91.

[44] Adler V, Yin Z, Fuchs SY, Benezra M, Rosario L, Tew KD, Pincus MR, Sardana M, Henderson CJ, Wolf CR, Davis RJ, Ronai Z. Regulation of JNK signaling by GSTp. EMBO J 1999;18:1321-34.

[45] Klatt P, Molina EP, De Lacoba MG, Padilla CA, Martinez-Galesteo E,
Barcena JA, Lamas S. Redox regulation of c-Jun DNA binding by reversible S-glutathiolation. FASEB J 1999;13:1481-90.

[46] Molina EP, Klatt P, Vazquez J, Marina A, de Lacoba MG, Perez-Sala D, Lamas S. Glutathionylation of the p50 subunit of NF-kappaB: a mechanism for redox-induced inhibition of DNA binding. Biochemistry 2001;40:14134-42.

[47] Wu HH, Thomas JA, Momand J. p53 protein oxidation in cultured cells in response to pyrrolidine dithiocarbamate: a novel method for relating the amount of p53 oxidation in vivo to the regulation of p53responsive genes. Biochem J 2000;35:87-93.

[48] Barrett WC, DeGnore JP, Konig S, Fales HM, Keng YF, Zhang ZY, Yim MB, Chock PB. Regulation of PTP1B via glutathionylation of the active site cysteine 215. Biochemistry 1999;38:6699-705.

[49] Denu JM, Dixon JE. Protein tyrosine phosphatases: mechanisms of catalysis and regulation. Curr Opin Chem Biol 1998;2:633-41.

[50] Mahadev K, Zilbering A, Zhu L, Goldstein BJ. Insulin-stimulated hydrogen peroxide reversibly inhibits protein-tyrosine phosphatase $1 \mathrm{~b}$ in vivo and enhances the early insulin action cascade. J Biol Chem 2001;276:21938-42.

[51] Babior BM, Lambeth JD, Nauseef W. The neutrophil NADPH oxidase. Arch Biochem Biophys 2002;397:342-4.

[52] Perego P, Paolicchi A, Tongiani R, Pompella A, Tonarelli P, Carenini $\mathrm{N}$, Romanelli S, Zunino F. The cell-specific anti-proliferative effect of reduced glutathione is mediated by gamma-glutamyl transpeptidasedependent extracellular pro-oxidant reactions. Int $\mathbf{J}$ Cancer 1997;71:246-50.

[53] Perego P, Gatti L, Carenini N, Dal Bo L, Zunino F. Apoptosis induced by extracellular glutathione is mediated by $\mathrm{H}(2) \mathrm{O}(2)$ production and DNA damage. Int J Cancer 2000;87:343-8.

[54] Del Bello B, Paolicchi A, Comporti M, Pompella A, Maellaro E. Hydrogen peroxide produced during gamma-glutamyl transpeptidase activity is involved in prevention of apoptosis and maintenance of proliferation in U937 cells. FASEB J 1999;13:69-79.

[55] Sullivan DM, Wehr NB, Fergusson MM, Levine RL, Finkel T. Identification of oxidant-sensitive proteins: TNF-alpha induces protein glutathiolation. Biochemistry 2000;39:11121-8.

[56] Nebreda AR, Porrai A. p38 MAP kinases: beyond the stress response. Trends Biochem Sci 2000;25:257-60.

[57] Reglinski J, Hoey S, Smith WE, Sturrock RD. Cellular response to oxidative stress at sulfhydryl group receptor sites on the erythrocyte membrane. J Biol Chem 1988;263:12360-6.

[58] Ciriolo MR, Paci M, Sette M, De Martino A, Bozzi A, Rotilio G. Transduction of reducing power across the plasma membrane by reduced glutathione. A $1 \mathrm{H}-\mathrm{NMR}$ spin-echo study of intact human erythrocytes. Eur J Biochem 1993;215:711-8.

[59] Filomeni G, Aquilano K, Rotilio G, De Martino A, Ciriolo MR. Glutathione disulfide and redox signalling events in U937 promonocytic cell line. In: Diederich M, editor. Proceedings of Signal Transduction Pathways and Regulation of Gene Expression as Therapeutic Targets, 2002 Jan 26-29; Kirchberg-Luxembourg.

[60] Daniel PT, Wieder T, Sturm I, Schulze-Osthoff K. The kiss of death: promises and failures of death receptors and ligands in cancer therapy. Leukemia 2001;15:1022-32.

[61] Angelini G, Gardella S, Ardy M, Ciriolo MR, Filomeni G, Di Trapani G, Clarke F, Sitia R, Rubartelli A. Antigen-presenting dendritic cells provide the reducing extracellular microenvironment required for $\mathrm{T}$ lymphocyte activation. Proc Natl Acad Sci U S A 2002;99:1491-6.

[62] Edinger AL, Thompson CB. Antigen-presenting cells control T cell proliferation by regulating amino acid availability. Proc Natl Acad Sci U S A 2002;99:1107-9. 